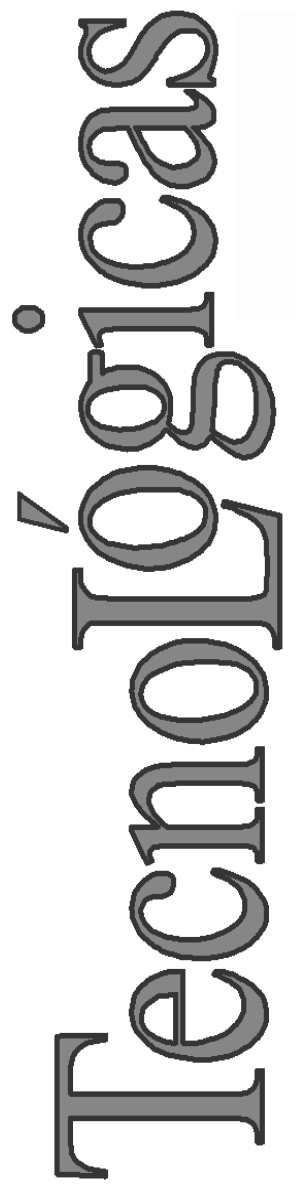

\title{
Compensación de Dispersión Cromática Usando Fibras de Cristal Fotónico con Distribución Hexagonal
}

\section{Chromatic Dispersion Compensation Using Photonic Crystal Fibers with Hexagonal Distribution}

Erick E. Reyes-Vera ${ }^{1}$ Nelson D. Gómez-Cardona ${ }^{2}$

1 Departamento de electrónica y telecomunicaciones, Facultad de ingenierías, Instituto Tecnológico Metropolitano, Medellín-Colombia erickreyes@itm.edu.co

2 Departamento de electrónica y telecomunicaciones, Facultad de ingenierías, Instituto Tecnológico Metropolitano, Medellín-Colombia nelsongomez@itm.edu.co 


\section{Resumen}

En el presente trabajo se muestran varias configuraciones de una de cristal fotónico con huecos distribuidos de forma hexagonal para la compensación de dispersión cromática en enlaces de comunicaciones ópticas. Para el análisis de las fibras se usó el método de elementos finitos vectorial con frontera dispersiva. A partir de estos resultados se estimó la variación de la dispersión y la pendiente de la dispersión con respecto al cambio del diámetro de los agujeros de la microestructura. Con lo anterior fue posible obtener valores de dispersión en las bandas C y L de las telecomunicaciones cercanos a los $-850 \mathrm{ps} / \mathrm{nm}^{*} \mathrm{~km}$, con pérdidas de confinamiento del orden de 10-3 dB/km.

\section{Palabras clave}

Dispersión cromática; fibra de cristal fotónico; telecomunicaciones; método de los elementos finitos.

\section{Abstract}

In this paper we show various configurations of photonic crystal fiber with hexagonal holes distribution for compensation of chromatic dispersion in optical communications links. The vectorial finite element method with scattering boundary condition was used for the analysis of the fibers. From these results it was estimated variation of the dispersion and the dispersion slope with respect to change in the diameter of the holes in the microstructure. With the above was possible to obtain values of dispersion in the $\mathrm{C}$ and $\mathrm{L}$ bands of telecommunications close to $-850 \mathrm{ps} /$ $\mathrm{nm}{ }^{*} \mathrm{~km}$, with confinement losses 10-3 dB / km.

\section{Keywords}

Chromatic dispersion; photonic crystal fiber; telecommunications; finite element method. 


\section{INTRODUCCIÓN}

Las principales causas de las limitaciones de transmisión de los sistemas ópticos de largo alcance a altas tasas de bits son la dispersión cromática (CD) y la dispersión por modos de polarización (PMD). Ambos fenómenos generan ensanchamiento temporal de los pulsos, en el primer caso, esto se debe a la dependencia del índice de refracción del material y del índice efectivo del modo de la luz que viaja por la fibra con la longitud de onda (dispersión del material y dispersión de la guía de onda). En el segundo caso, la birrefringencia intrínseca ó inducida en la fibra óptica genera un retraso temporal entre los modos con polarizaciones ortogonales (Govind P., 2002).

La CD puede ser pre-compensada, post compensada o una combinación de las dos anteriores (Hui \& O'Sullivan, 2009), esto significa que, los dispositivos de compensación de dispersión (DCD) pueden estar justo después de trasmisor pero antes de entra a la fibra (pre) y/o después de la fibra pero antes del detector (post). De igual forma los DCD pueden dividirse en dos grandes grupos: Compensación Electrónica de Dispersión (EDC) y Compensación Óptica de Dispersión (ODC). En el primero de estos grupos utiliza técnicas de ecualización y procesamiento de señales para eliminar la dispersión cromática acumulada al final del enlace (Rafique \& Ellis, 2011), (McGhan et al., 2008), (Cui et al., 2011). La principal característica de este tipo de compensadores de dispersión es que permite, en los sistemas WDM, hacer una compensación independiente para cada canal (La EDC está definida en la recomendación ITU G.959.1). En el segundo grupo se ubican las técnicas basadas en fenómenos ópticos, lineales o no lineales, en estos se utilizan fibras ópticas con dispersión negativa, conocidas en este contexto como fibras para compensar dispersión (DCF) y redes de Bragg "chirpadas" (Spolitis \& Ivanovs, 2011), (Fadhil et al., 2011). Como en estas fibras la compensación es óptica entonces no se generan retrasos adicionales, (cosa que si ocurre cuando se hace conversión óptica/electrónica y procesamiento digital de señales).

Una de las técnicas ópticas más promisorias para la compensación de dispersión, ya sea cromática, es el uso de fibras ópticas de 
cristal fotónico (PCF) (Janrao \& Janyani, 2011; Johny et al., 2012; Li et al., 2011; Lucki, 2011; Malheiros-silveira et al., 2011). Estas fibras se construyen a partir de micro-estructuras huecas las cuales no poseen variaciones en la dirección longitudinal de la fibra. Así, podemos imaginar este tipo de fibras como un cilindro con muchos huecos alrededor de un núcleo sólido, el diámetro de estos huecos y su distancia de separación son del orden micro-métrico o sub-micrométrico (del orden de magnitud de la longitud de onda que viaja por la fibra) por lo cual la propagación de la luz se hace a través de algunos de estos mecanismos: índice efectivo o bandafotónica prohibida (An, 2012). Estos mecanismos de propagación unidos a la asimetría radial de las fibras de cristal fotónico hacen que en estas se presenten fenómenos que en las fibras ópticas estándar no es posible observar, como por ejemplo: gran área modal, dispersión plana (es decir pendiente de dispersión igual a cero), dispersión negativa y alta birrefringencia, entre otros (Méndez \& Morse, 2007). En particular, las PCF con dispersión negativa en la región de las bandas $\mathrm{C}$ y L de las telecomunicaciones son usadas para la compensación de CD.

\section{METOdOLOGíA}

Los modos de propagación electromagnéticos de la microestructura se encuentran solucionando la ecuación de onda vectorial,

$$
\nabla \times \varepsilon^{-1} \nabla \times \mathbf{H}=k_{0}^{2} \mathbf{H}
$$

En la ecuación anterior, $\mathbf{H}$ es el campo magnético, $\varepsilon$ es permitividad dieléctrica del medio, $k_{0}=2 \pi / \lambda$, es el vector de onda, $\lambda$ es la longitud de onda en el vacío. Para solucionar la ecuación anterior se usó el método de elementos finitos Scattering Boundary Condition.

El procedimiento anterior permite encontrar el índice de propagación efectivo, $n_{\text {eff, }} \mathrm{y}$ la distribución espacial del modo fundamental un rango de longitudes de onda entre 500 y $1600 \mathrm{~nm}$. Los valores calculados de $n_{\text {eff }}$ son usados para estimar el coeficiente de 
dispersión $D$ [ps/nm $\mathrm{km}$ ], y el factor de pérdidas por confinamiento $L_{c}[\mathrm{~dB} / \mathrm{km}]$, los cuales vienen dados por las expresiones,

$$
D=-\frac{\lambda}{c} \frac{\partial^{2} \operatorname{Re}\left\{n_{e f f}\right\}}{\partial^{2} \lambda}
$$

$L_{c}=8.686 \operatorname{Im}\left\{n_{e f f}\right\}$

$\lambda$ es la longitud de onda, y $c$ la velocidad de la luz en el vacío. La pendiente de la dispersión definida como la derivada de $\mathrm{D}$ con respecto a $\lambda, S=\partial D / \partial \lambda$, fue también calculada.

Para una compensación adecuada de la dispersión cromática es necesario que las longitudes $L$ de las fibras, sus coeficientes de dispersión $D$ y las pendientes de la dispersión $S$, cumplan con las siguientes condiciones,

$L_{S M F} D_{S M F}=L_{P C F} D_{P C F}$

$L_{S M F} S_{S M F}=L_{P C F} S_{P C F}$

Los subscriptos SMF y PCF hacen referencia a la fibra monomodo estándar y a la fibra de cristal fotónico, respectivamente. Combinado las ecuaciones anteriores es posible definir un parámetro adicional conocido como Pendiente de Dispersión Relativa (RSD),

$R S D=\frac{D_{S M F}}{S_{S M F}}=\frac{-D_{P C F}}{-S_{P C F}}$

Las Fig. 1(a) y 1(b) muestran las secciones transversales de la PCF y la fibra SMF analizadas. La ecuación de Sellmeier fue usada para modelar el índice de refracción de la sílice. 


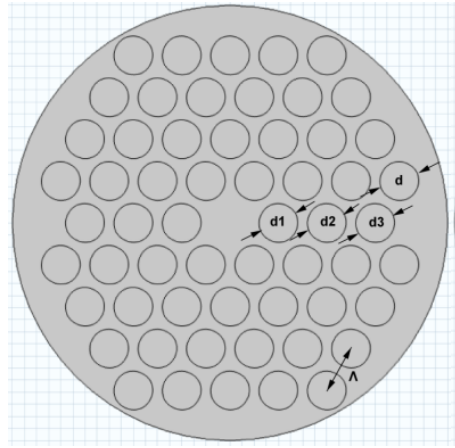

(a)

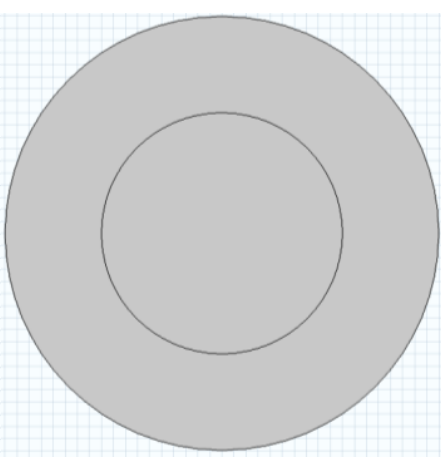

(b)

Fig. 1. Sección transversal de las fibras analizadas en este trabajo (a) Fibra de cristal fotonico PCF y (b) Fibra SMF 28

\section{RESULTADOS Y DISCUSIÓN}

La propagación de la luz en las PCF es similar a las fibras ópticas estándares, es decir, a través del fenómeno de reflexión total interna, sin embargo, parámetros de la propagación de la luz, tales como la dispersión y el índice efectivo tienen una fuerte dependencia con el periodo de la microestructura $\square$ y el diámetro de los agujeros $d_{i}$. Se analizaron seis configuraciones totalmente diferentes con el fin de observar el comportamiento de la dispersión al variar el perfil de la microestructura y así establecer de estas configuraciones cual es más adecuada para compensar la dispersión cromática en un enlace de telecomunicaciones. Se realizó la simulación manteniendo constante el periodo de la microestructura $\Lambda=0.8 \mu \mathrm{m}$ y el diámetro de los agujeros del cuarto anillo $\mathrm{d}=0.64 \mu \mathrm{m}$. Los diámetros $\mathrm{d}_{1}, \mathrm{~d}_{2} \mathrm{y} \mathrm{d}_{3}$, correspondientes a los diámetros de los agujeros del primer, segundo y tercer anillo, respectivamente, se variaron como se muestra en la Tabla 1.

Tabla 1. Parámetros geométricos de las $\mathrm{PCF}^{\prime}$ s analizadas

\begin{tabular}{ccccccc}
\hline Parámetro & PCF A & PCF B & PCF C & PCF D & PCF E & PCF F \\
\hline $\mathrm{d} 1[\mu \mathrm{m}]$ & 0.64 & 0.34 & 0.64 & 0.34 & 0.34 & 0.34 \\
$\mathrm{~d} 2[\mu \mathrm{m}]$ & 0.64 & 0.64 & 0.64 & 0.64 & 0.34 & 0.34 \\
$\mathrm{~d} 3[\mu \mathrm{m}]$ & 0.64 & 0.64 & 0.34 & 0.34 & 0.34 & 0.64 \\
\hline
\end{tabular}


Los resultados obtenidos a través del método de elementos finitos se ilustran en las Fig. 2(a) y 2(b), donde claramente se observa que a medida que se incrementa la longitud de onda, el valor de índice efectivo de las configuraciones analizadas disminuye, esto se debe principalmente a que a pequeñas longitudes de onda la luz que se propaga por la fibra está más confinada en el centro de la microestructura. Es por esta razón que las pérdidas por confinamiento, obtenidas de la ecuación 2(b) muestran perdidas nulas a bajas longitudes de onda. Además, es fácil observar como las configuraciones PCF C y PCF E son las que presentan mayores pérdidas por confinamiento en la región de telecomunicaciones, esto se debe principalmente a que al variar $\mathrm{d}_{3}$ está generando que la luz con longitudes de onda en las bandas C y L no esté bien confinada en el núcleo de la fibra. Lo anterior contrasta fuertemente con las configuraciones PCF B y PCF F, las cuales presentan perdidas casi nulas en el rango analizado. Lo anterior se debe a que estas configuraciones poseen un núcleo más grande, además los anillos 3 y 4 ayudan a mejorar el confinamiento de la luz a través del núcleo.

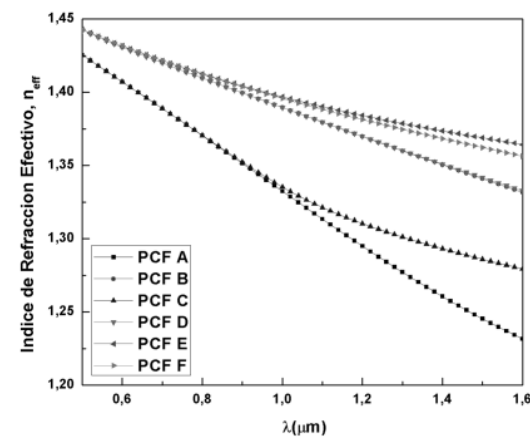

(a)

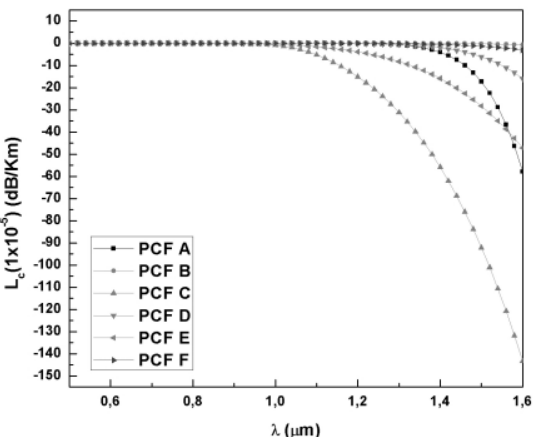

(b)

Fig. 2. (a) Índice efectivo $n_{\text {eff }} \mathrm{y}(\mathrm{b})$ factor de pérdidas por confinamiento $L_{c}$ para las diferentes configuraciones de PCF's

A través de (2a) y empleando los resultados obtenidos en COMSOL para los valores de $n_{\text {eff, }}$, se calcularon las curvas de dispersión para las diferentes configuraciones, tal como se muestra en la Fig. 3(a). De los resultados obtenidos, y mostrados en la Fig. 3(a), podemos ver que, aunque las configuraciones PCF C, PCF E y 
PCF F tienen valores negativos del parámetro de dispersión $D$ en la región de telecomunicaciones, el valor de la pendiente de dispersión $S$, en esta misma región, tiene valores positivos, como se observa en la figura 3(b) y por lo tanto no cumplen con la condición impuesta por la ecuación 5, la cual exige que la $R D S$ sea positiva, condición que se cumple solo cuando ambos parámetros tienen signo negativo. De otro lado, y tal como se observa en las Fig. 3(a) y 3(b) las configuraciones PCF A, PCF B y PCF D tienen valores negativos para ambos parámetros $D$ y $S$ y por lo tanto cumplen con la condición impuesta en la ecuación 5, mostrando que empleando alguna de estas configuraciones es posible compensar la dispersión cromática que se presenta en la fibra SMF 28. Aunque el valor de $R D S$ debe ser positivo, tal cual se muestra en la Fig. 4, se debe cumplir que los parámetros $D$ y $S$ tengan valores negativos para poder compensar los efectos de la dispersión cromática causados en la fibra SMF-28.
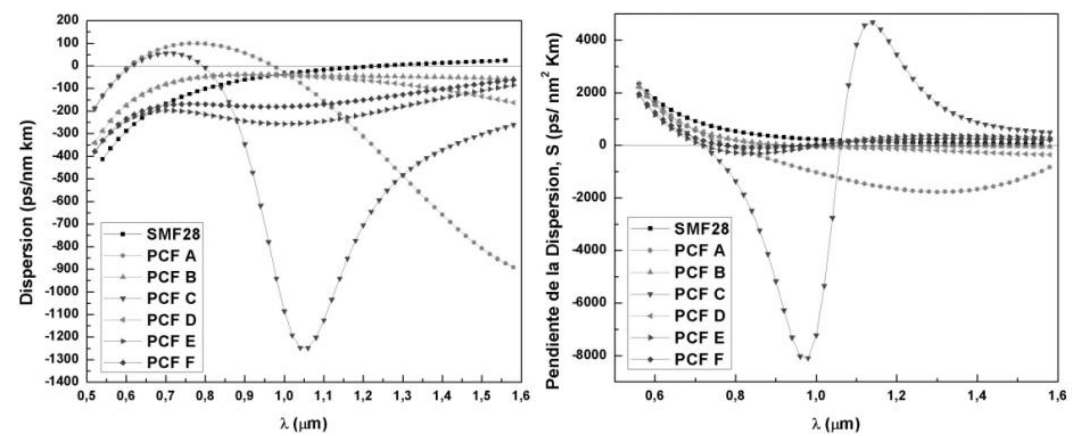

Fig. 3. (a) Dispersión $D$ y (b) Pendiente de la dispersión $S$ para las diferentes configuraciones de PCF's

En la Tabla 2 se muestran, a manera de ejemplo, que longitud de PCF se requiere para compensar la dispersión causada en $100 \mathrm{~km}$ de fibra SMF-28, y las pérdidas máximas en $\mathrm{dB}$, para las algunos valores de longitud de onda en las bandas $\mathrm{C}$ y L. 
Tabla 2. Longitud de fibra LPCF y pérdidas por confinamiento Lc para compensar la dispersión cromática en 100km de fibra SMF-28

\begin{tabular}{ccccc}
\hline$\lambda[\mathrm{um}]$ & $\begin{array}{c}\text { LPCF A } \\
{[\mathrm{km}]}\end{array}$ & $\begin{array}{c}\text { LPCF B } \\
{[\mathrm{km}]}\end{array}$ & $\begin{array}{c}\text { LPCF } \mathrm{D} \\
{[\mathrm{km}]}\end{array}$ & $\begin{array}{c}\mathrm{L}_{\mathrm{C}} \text { Max. } \\
{[\mathrm{dB}]}\end{array}$ \\
\hline 1.540 & 2.57 & 2.64 & 2.71 & $\sim 1.610-3$ \\
1.560 & 39.6 & 40.9 & 42.0 & $\sim 0.0$ \\
1.580 & 14.79 & 14.86 & 14.89 & $\sim 2.810-3$ \\
\hline
\end{tabular}

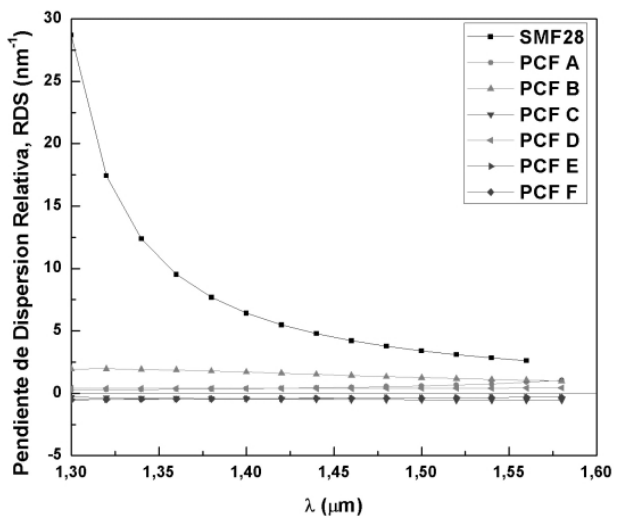

Fig. 4. $R D S$ de la fibra después de la compensación empleando las diferentes PCF propuestas en el trabajo

\section{CONCLUSIONES}

Se evidencio que empleando fibras ópticas micro estructuradas pueden ser diseñados dispositivos ópticos integrados para la compensación de la dispersión en enlaces de telecomunicaciones, con el fin de mejorar tanto la calidad de señal como la distancia que se puede alcanzar sin necesidad de realizar grandes cambios en la infraestructura ya instalada.

Se pudo observar la importancia de los parámetros de la mircroestructura, específicamente en este trabajo se evaluó el cambio en el diámetro de los agujeros; observamos que variar el diámetro de los agujeros de uno de los anillos altera el comportamiento de la dispersión en estas fibras. Mostrando de esta manera una versatilidad y un amplio potencial de aplicaciones de este tipo de fibras para optimizar los sistemas de comunicaciones a fibra óptica. 
Alterando la geometría de estas fibras ópticas microestructuradas, se puede llegar a obtener sistemas con baja dispersión como lo muestran las configuraciones PCF A, PCF B, y PCF D, además de permitir mayores distancias de propagación ya que tanto sus pérdidas en las bandas $\mathrm{C}$ y L son muy bajas. A través de la alteración de los parámetros se puede manipular la longitud de onda a la cual desearía realizar compensación de dispersión del sistema de comunicaciones, permitiendo sistemas versátiles.

\section{AGRADECIMIENTOS}

Los autores agradecen a la dirección de investigaciones del Instituto Tecnológico Metropolitano por su apoyo a través del proyecto Parque i

\section{REFERENCIAS}

An, A. (2012). Design of a Polarization-Maintaining Equiangular Spiral Photonic Crystal Fiber for Residual Dispersion Compensation Over, 24(11), 930-932.

Cui, Y., Xu, K., Dai, J., Dai, Y., Wu, J., \& Lin, J. (2011). Compensation of the chromatic dispersion in long-reach analog optical links incorporating parallel electro-optic phase and intensity modulators. 2011 International Topical Meeting on Microwave Photonics jointly held with the 2011 AsiaPacific Microwave Photonics Conference, 270-273.

Fadhil, H. a, Ahmed, H. Y., \& Aljunid, S. a. (2011). Performance analysis of hybrid optical OFDM system with high order dispersion compensation. The 17th Asia Pacific Conference on Communications, (October), $117-120$.

Govind P., A. (2002). Fiber-Optic Communications Systems, Third Edition. (Third Edit., Vol. 6, pp. 0-471). John Wiley \& Sons.

Hui, R., \& O'Sullivan, M. (2009). Fiber optic measurement techniques. Academic Press. Retrieved from

Janrao, N., \& Janyani, V. (2011). Dispersion compensation fiber using square hole PCF. 2011 International Conference on Communications and Signal Processing, 436-438.

Johny, J., Shashidharan, S., Sudheer, S. K., \& Kumar, K. S. (2012). Design and Simulation of a Radio Over Fiber System with Chromatic Dispersion and Polarisation Mode Dispersion Compensation, 1-4. 
Li, J., Wang, R., Wang, J., Xu, Z., \& Su, Y. (2011). Novel large negative dispersion photonic crystal fiber for dispersion compensation. 2011 Second International Conference on Mechanic Automation and Control Engineering, 1443-1446.

Lucki, M. (2011). Optimization of microstructured fiber for dispersion compensation purposes. 2011 13th International Conference on Transparent Optical Networks, 1-4.

Malheiros-silveira, G. N., Jr, J. A. M., Hernández-figueroa, H. E., \& Member, S. (2011). Improving Dispersion Compensation of Photonic Crystal Fibers Through Air Hole Core Insertion, 121-124.

McGhan, D., O’Sullivan, M., Sotoodeh, M., Savchenko, a, Bontu, C., Belanger, M., \& Roberts, K. (2008). Electronic dispersion compensation. 2006 Optical Fiber Communication Conference and the National Fiber Optic Engineers Conference, 25(1), 158-167.

Rafique, D., \& Ellis, A. D. (2011). Various Nonlinearity Mitigation Techniques Employing Optical and Electronic Approaches. IEEE Photonics Technology Letters, 23(23), 1838-

Spolitis, S., \& Ivanovs, G. (2011). Extending the reach of DWDM-PON access network using chromatic dispersion compensation. 2011 IEEE Swedish Communication Technologies Workshop SweCTW, 29-33. 